\title{
CONVERGENCE VERSUS INTEGRABILITY IN POINCARÉ-DULAC NORMAL FORM
}

\author{
NGuyen Tien Zung
}

\begin{abstract}
We show that, to find a Poincaré-Dulac normalization for a vector field is the same as to find and linearize a torus action which preserves the vector field. Using this toric characterization and other geometrical arguments, we prove that any local analytic vector field which is integrable in the non-Hamiltonian sense admits a local analytic Poincaré-Dulac normalization. These results generalize the main results of our previous paper [12] from the Hamiltonian case to the nonHamiltonian case. Similar results are presented for the case of isochore vector fields.
\end{abstract}

\section{Introduction}

One of the classical problems in ordinary differential equations and dynamical systems is to find necessary and sufficient conditions for the existence of a local analytic (i.e. convergent) Poincaré-Dulac normalization for an analytic vector field near an equilibrium point. Roughly speaking, there are (at least) two approaches to this problem: analytical and geometrical. In the analytical approach, with classical results due to Poincaré, Siegel, Bruno and others, one uses Diophantine conditions to control small divisors which appear in a stepby-step normalization process, and uses the fast converging iteration method to arrive at convergence results, see e.g. $[3,8]$. In the geometrical approach, one replaces Diophantine conditions by symmetries and first integrals, and replaces the fast convergence method by arguments of geometrical nature. Some recent papers on the subject like $[1,4,5,9,10]$ contain some geometrical ingredients (commuting vector fields, symmetry groups), but the methods used remain mostly analytical. In the present paper, we will follow the geometrical approach in a more substantial way. We will use torus actions to characterize Poincaré-Dulac normalization, and use this characterization to show that any analytic vector field which is integrable in the non-Hamiltonian sense admits a convergent Poincaré-Dulac normalization. More precisely, we have the following

Theorem 1.1. Let $X$ be a local analytic vector field in $\left(\mathbb{K}^{n}, 0\right)$, where $\mathbb{K}=\mathbb{R}$ or $\mathbb{C}$, with $X(0)=0$. Suppose that there is a natural number $m, 1 \leq m \leq n$,

Received May 23, 2001.

Revised version received March 13, 2002.

2000 Mathematics Subject Classification. 37G05, 70K45, 34C14, 70GXX.

Key words and phrases. Poincaré-Dulac normal form, torus action, integrability, commuting vector fields, isochore vector fields. 
such that there are $m$ local analytic vector fields $X_{1}=X, X_{2}, \ldots, X_{m}$ and $n-m$ local analytic functions $f_{1}, \ldots, f_{n-m}$ in $\left(\mathbb{K}^{n}, 0\right)$ with the following properties:

i) The vector fields $X_{1}=X, X_{2}, \ldots, X_{m}$ commute pairwise, i.e.

$$
\left[X_{i}, X_{j}\right]=0 \quad \forall i, j=1, \ldots, m,
$$

and they are linearly independent almost everywhere, i.e.

$$
X_{1} \wedge \ldots \wedge X_{m} \neq 0 \text {. }
$$

ii) The functions $f_{1}, \ldots, f_{n-m}$ are common first integrals for $X_{1}, \ldots, X_{m}$, i.e.

$$
X_{i}\left(f_{j}\right)=0 \quad \forall i=1, \ldots, m ; j=1, \ldots, n-m,
$$

and they are functionally independent almost everywhere, i.e.

$$
d f_{1} \wedge \ldots \wedge d f_{n-m} \neq 0 \text {. }
$$

Then there exists a local analytic Poincaré-Dulac normalization for $X$ in a neighborhood of 0 in $\mathbb{K}^{n}$. In other words, there is a local analytic system of coordinates in $\left(\mathbb{K}^{n}, 0\right)$ such that if we denote by $X^{s}$ the semi-simple part of the linear part of $X$ with respect to this coordinate system then we have

$$
\left[X, X^{s}\right]=0 .
$$

\section{Remarks.}

1. In the above theorem, $m$ can be any natural number between 1 and $n$. When $m=1$, we have a complete set of first integrals (and the vector field will be automatically very resonant if $n>1$ ). When $m=n$ we have a complete set of commuting infinitesimal symmetries. When $1<m<n$, we have a mixture of commuting infinitesimal symmetries and first integrals.

2. If a vector field satisfies the two conditions i) and ii) of the above theorem, then one says that $X$ is integrable in the non-Hamiltonian sense (see e.g. $[2,13]$ ). The above theorem may viewed as the non-Hamiltonian version of Theorem 1.1 of our previous paper [12]. Our proof of the above theorem is also based on a geometrical method developed in [12].

3. When $n=2$ the above theorem has been obtained by Bruno and Walcher [4]. Their proof is based on Bruno's results [3], and therefore uses the fast convergence method directly or indirectly, in contrast to our method.

4. It is interesting to compare the above theorem to similar but different results of Stolovitch $[9,10]$. The similarity: Stolovitch's results are also about the existence of a convergent normalization under some integrability conditions. The difference: Stolovitch needs only formal first integrals (it is roughly speaking the $A$-condition of Bruno, see $[3,9]$ ) while we use analytic (or eventually meromorphic - we will consider the meromorphic case in a subsequent paper) ones, but to compensate for this he also needs Diophantine conditions while we don't.

The reason why we are interested in integrability conditions while studying local normal forms of vector fields is the following: both the search for first integrals and symmetries and the search for a normalization are methods to 
reduce and solve differential equations, and in good cases one can solve the equations completely by both methods, so it is natural that the two methods should be very closely related. Theorem 1.1, as well as the title of the present paper, is about such relations. (See [12] for a more detailed discussion in the Hamiltonian case).

The rest of this paper is organized as follows: In Section 2 we will use torus actions to characterize Poincaré-Dulac normalizations (see Proposition 2.1). As a corollary of this characterization, we obtain that a real analytic vector field admits a local real analytic Poincaré-Dulac normalization if and only if it admits a local holomorphic Poincaré-Dulac normalization (see Proposition 2.2). We will prove Theorem 1.1 in Section 3, using the above toric characterization and a geometrical method of approximation developed in [12]. In Section 4 we will extend our results to the case of isochore (i.e. volume preserving) vector fields. In particular, we will obtain an improvement of a result of Vey [11] about the existence of a simultaneous normalization for an $(n-1)$-tuple of pairwise commuting holomorphic isochore vector fields in $\left(\mathbb{C}^{n}, 0\right)$ : we will show that, without any nonresonance/nondegeneracy condition, Vey's theorem is still true, both in $\left(\mathbb{C}^{n}, 0\right)$ and in $\left(\mathbb{R}^{n}, 0\right)$.

\section{Normalization and torus action}

In this section, we will recall some basic notions about Poincaré-Dulac normal forms, and show that these normal forms are governed by torus actions.

Let $X$ be a given analytic vector field in a neighborhood of 0 in $\mathbb{K}^{n}$, where $\mathbb{K}=\mathbb{R}$ or $\mathbb{C}$, with $X(0)=0$. When $\mathbb{K}=\mathbb{R}$, we may also view $X$ as a holomorphic (i.e. complex analytic) vector field by complexifying it. Denote by

$$
X=X^{(1)}+X^{(2)}+X^{(3)}+\ldots
$$

the Taylor expansion of $X$ in some local system of coordinates, where $X^{(k)}$ is a homogeneous vector field of degree $k$ for each $k \geq 1$. The algebra of linear vector fields on $\mathbb{K}^{n}$, under the standard Lie bracket, is nothing but the reductive algebra $g l(n, \mathbb{K})=\operatorname{sl}(n, \mathbb{K}) \oplus \mathbb{K}$. In particular, we have

$$
X^{(1)}=X^{s}+X^{n i l},
$$

where $X^{s}$ (resp., $X^{\text {nil }}$ ) denotes the semi-simple (resp., nilpotent) part of $X^{(1)}$. There is a complex linear system of coordinates $\left(x_{j}\right)$ in $\mathbb{C}^{n}$ which puts $X^{s}$ into diagonal form:

$$
X^{s}=\sum_{j=1}^{n} \gamma_{j} x_{j} \partial / \partial x_{j},
$$

where $\gamma_{j}$ are complex coefficients, called eigenvalues of $X$ (or $X^{(1)}$ ) at 0 .

For each natural number $k \geq 1$, the vector field $X^{s}$ acts linearly on the space of homogeneous vector fields of degree $k$ by the Lie bracket, and the monomial 
vector fields are the eigenvectors of this action:

$$
\left[\sum_{j=1}^{n} \gamma_{j} x_{j} \partial / \partial x_{j}, x_{1}^{b_{1}} x_{2}^{b_{2}} \ldots x_{n}^{b_{n}} \partial / \partial x_{l}\right]=\left(\sum_{j=1}^{n} b_{j} \gamma_{j}-\gamma_{l}\right) x_{1}^{b_{1}} x_{2}^{b_{2}} \ldots x_{n}^{b_{n}} \partial / \partial x_{l} .
$$

When an equality of the type

$$
\sum_{j=1}^{n} b_{j} \gamma_{j}-\gamma_{l}=0
$$

holds for some nonnegative integer $n$-tuple $\left(b_{j}\right)$ with $\sum b_{j} \geq 2$, we will say that the monomial vector field $x_{1}^{b_{1}} x_{2}^{b_{2}} \ldots x_{n}^{b_{n}} \partial / \partial x_{l}$ is a resonant term, and that the $n$-tuple $\left(b_{1}, \ldots, b_{l}-1, \ldots, b_{n}\right)$ is a resonance relation for the eigenvalues $\left(\gamma_{i}\right)$. More precisely, a resonance relation for the $n$-tuple of eigenvalues $\left(\gamma_{j}\right)$ of a vector field $X$ is an $n$-tuple $\left(c_{j}\right)$ of integers satisfying the relation $\sum c_{j} \gamma_{j}=0$, such that $c_{j} \geq-1, \sum c_{j} \geq 1$, and at most one of the $c_{j}$ may be negative. Notice here a major difference between the non-Hamiltonian case (which is the case of this paper) and the Hamiltonian case (studied in [12]) : in the Hamiltonian case there are no positivity conditions like $c_{j} \geq-1$, so in the Hamiltonian case the set of resonance relations for a given $n$-tuple of frequencies is a sublattice in $\mathbb{Z}^{n}$, while in the non-Hamiltonian case the set of resonance relations for a given $n$-tuple of eigenvalues is a convex set in $\mathbb{Z}^{n}$ which does not contain any line.

Denote by

$$
\mathcal{R} \subset \mathbb{Z}^{n}
$$

the subset of $\mathbb{Z}^{n}$ consisting of all resonance relations $\left(c_{j}\right)$ for a given vector field $X$. The number

$$
q=\operatorname{dim}_{\mathbb{Z}}\left(\text { linear hull of } \mathcal{R} \text { in } \mathbb{Z}^{\mathrm{n}}\right)
$$

is called the degree of resonance of $X$. Of course, the degree of resonance depends only on the eigenvalues of the linear part of $X$, and does not depend on the choice of local coordinates. If $q=0$ then we say that $X$ is nonresonant.

The vector field $X$ is said to be in Poincaré-Dulac normal form (or normal form for short) if it commutes with the semisimple part of its linear part (see e.g. $[3,8])$ :

$$
\left[X, X^{s}\right]=0 .
$$

The above equation means that if $X$ is in normal form then its nonlinear terms are resonant. In particular, if $X$ is nonresonant then there are no resonant terms, and $X$ is linear when it is in normal form. A transformation of coordinates which puts $X$ in Poincaré-Dulac normal form is called a Poincaré-Dulac normalization. It is a classical result of Poincaré and Dulac that any analytic vector field which vanishes at 0 admits a formal Poincaré-Dulac normalization. 
Denote by $\mathcal{Q} \subset \mathbb{Z}^{n}$ the integral sublattice of $\mathbb{Z}^{n}$ consisting of $n$-dimensional vectors $\left(\rho_{j}\right) \in \mathbb{Z}^{n}$ which satisfy the following properties:

$$
\sum_{j=1}^{n} \rho_{j} c_{j}=0 \forall\left(c_{j}\right) \in \mathcal{R}, \text { and } \rho_{j}=\rho_{k} \text { if } \gamma_{j}=\gamma_{k}
$$

(where $\mathcal{R}$ is the set of resonance relations for the eigenvalues $\left(\gamma_{j}\right)$ as before). We will call the number

$$
r=\operatorname{dim}_{\mathbb{Z}} \mathcal{Q}
$$

the toric degree of $X$ (or $X^{(1)}$ ). Of course, this number depends only on the eigenvalues of the linear part of $X$, and we have the following inequality:

$$
q+r \leq n
$$

where $q$ is the degree of resonance.

Let $\left(\rho_{j}^{1}\right), \ldots,\left(\rho_{j}^{r}\right)$ be a basis of $\mathcal{Q}$. For each $k=1, \ldots, r$ define the following diagonal linear vector field $Z_{k}$ :

$$
Z_{k}=\sum_{j=1}^{n} \rho_{j}^{k} x_{j} \partial / \partial x_{j}
$$

The vector fields $Z_{1}, \ldots, Z_{r}$ have the following remarkable properties:

a) They commute pairwise and commute with $X^{s}$ and $X^{\text {nil }}$, and they are linearly independent almost everywhere.

b) $i Z_{j}$ is a periodic vector field of period $2 \pi$ for each $j \leq r$ (here $i=\sqrt{-1}$ ). What does it mean is that if we write $i Z_{j}=\Re\left(i Z_{j}\right)+i \Im\left(i Z_{j}\right)$, then $\Re\left(i Z_{j}\right)$ is a periodic real vector field in $\mathbb{C}^{n}=\mathbb{R}^{2 n}$ which preserves the complex structure.

c) Together, $i Z_{1}, \ldots, i Z_{r}$ generate an effective linear $\mathbb{T}^{r}$-action in $\mathbb{C}^{n}$, which preserves $X^{s}$ and $X^{n i l}$.

If $X$ is in Poincaré-Dulac normal form, i.e. $\left[X, X^{s}\right]=0$, then we also have

$$
\left[X, Z_{k}\right]=0 \quad \forall k=1, \ldots, r .
$$

Indeed, if $W=x_{1}^{b_{1}} x_{2}^{b_{2}} \ldots x_{n}^{b_{n}} \partial / \partial x_{l}$ is a resonant monomial term, then we have $\sum b_{j} \gamma_{j}-\gamma_{l}=0$, which implies, via Equation (2.9), that $\sum_{j=1}^{n} b_{j} \rho_{j}^{k}-\rho_{l}=0$, and therefore $\left[Z_{k}, W\right]=\left(\sum_{j=1}^{n} b_{j} \rho_{j}^{k}-\rho_{l}\right) W=0$. Hence if all non-linear terms in $X$ are resonant then we have $\left[Z_{k}, X\right]=\left[Z_{k}, X^{(1)}\right]=0$.

The above commutation relations mean that if $X$ is in normal form, then it is preserved by the effective $r$-dimensional torus action generated by $i Z_{1}, \ldots, i Z_{r}$.

Conversely, suppose that there is a local analytic effective action of $\mathbb{T}^{r}$ in $\left(\mathbb{C}^{n}, 0\right)$ which preserves $X$, and whose linear part is generated by $i Z_{1}, \ldots, i Z_{r}$ (in other words, its weights are given by the lattice $\mathcal{Q})$. Then using the classical Bochner's linearization theorem for compact group actions (via the standard averaging method), we can linearize this torus action, i.e. we may suppose that the action is linear (in an appropriate local analytic coordinate system). Then this action is actually generated by $i Z_{1}, \ldots, i Z_{r}$. Since the action preserves $X$ by assumptions, we have that $\left[X, Z_{1}\right]=\ldots=\left[X, Z_{r}\right]=0$. But by definition of 
$Z_{1}, \ldots, Z_{r}$, the semisimple part of the linear part of $X$ is a linear combination of these vector fields $Z_{1}, \ldots, Z_{r}$, hence we have $\left[X, X^{s}\right]=0$. In other words, a linearization of our torus action is also a Poincaré-Dulac normalization for $X$. Thus we have proved the following result:

Proposition 2.1. A holomorphic vector field $X$ in a neighborhood of 0 in $\mathbb{C}^{n}$ admits a convergent Poincaré-Dulac normalization if and only if it is preserved by an effective holomorphic action of a real torus of dimension $r$, where $r$ is the toric degree of $X^{(1)}$ as defined in (2.10), in a neighborhood of 0 in $\mathbb{C}^{n}$, which has 0 as a fixed point and whose linear part at 0 has appropriate weights (given by the lattice $\mathcal{Q}$ defined in (2.9), which depends only on the linear part $X^{(1)}$ of $X)$.

Remark. The above proposition is true in the formal category as well. But of course, any vector field admits a formal Poincaré-Dulac normalization, and a formal torus action.

When $X$ is real analytic, the torus action in the above proposition still lives in the complex space in general. But in this case, there is a natural complex conjugation in the torus action, which allows us to deduce from Proposition 2.1 the following result:

Proposition 2.2. A real analytic vector field $X$ in a neighborhood of 0 in $\mathbb{R}^{n}$ with $X(0)=0$ admits a local real analytic Poincaré-Dulac normalization if and only if it admits a local holomorphic Poincaré-Dulac normalization when considered as a holomorphic vector field.

The proof of Proposition 2.2 is absolutely similar to the proof of Proposition 1.3 of our previous paper [12] (its Hamiltonian version), so we will omit it here.

\section{Torus actions for integrable vector fields}

Proof of Theorem 1.1. Invoking Proposition 2.1, we will prove Theorem 1.1 by finding a torus action. In view of Proposition 2.2, it suffices to prove Theorem 1.1 in the complex case, so in this section we will assume that $\mathbb{K}=\mathbb{C}$.

Recall that a vector field $X$ is said to be in normal form up to order $M$ (where $M$ is a natural number) in a given coordinate system if $\left[X, X^{s}\right]=O\left(|x|^{M}\right)$ (i.e. $\left[X, X^{s}\right]$ does not contain terms of order $\left.<M\right)$, where $X^{s}$ denotes the semisimple part of the linear part of $X$ in this coordinate system. A coordinate transformation that puts $X$ in normal form up to order $M$ is called a normalization up to order $M$. Such a local holomorphic normalization up to order $M$ always exists (for any $M$ ), according to the classical theorem of Poincaré and Dulac. We have the following lemma, whose proof is straightforward and uses only elementary linear algebra:

Lemma 3.1. Suppose that a vector field $X$ is in Poincaré-Dulac (formal or holomorphic) normal form. Denote by $r$ the toric degree of $X$, and by $Z_{1}, \ldots, Z_{r}$ the vector fields defined by Equation (2.12). If $Y$ is a vector field which commutes 
with $X$ then $\left[Z_{k}, Y\right]=0$ for $k=1, \ldots, r$. If $f$ is a first integral for $X$, i.e. $X(f)=0$, then $Z_{k}(f)=0$ for $k=1, \ldots, r$. If $X$ is in normal form up to order $M$ (for some natural number $M)$ then $\left[Z_{k}, Y\right]=O\left(|x|^{M}\right)$ and $Z_{k}(f)=O\left(|x|^{M}\right)$ for $k=1, \ldots, r$.

Now let $X$ be a local holomorphic vector field in $\left(\mathbb{C}^{n}, 0\right)$ which satisfies the conditions of Theorem 1.1, with $X_{1}=X, \ldots, X_{m}$ and $f_{1}, \ldots, f_{n-m}$ being the $m$ local holomorphic pairwise commuting vector fields and $(n-m)$ holomorphic common first integrals respectively. By assumptions, $X_{1} \wedge \ldots \wedge X_{m} \neq 0$ and $d f_{1} \wedge \ldots \wedge d f_{n-m} \neq 0$ almost everywhere.

Fix a holomorphic coordinate system $x=\left(x_{j}\right)$ in $\mathbb{C}^{n}$, a standard Hermitian metric in $\mathbb{C}^{n}$ which goes with it, and a sufficiently small positive number $\epsilon_{0}$. Denote by

$S=\left\{x \in \mathbb{C}^{n},|x|<\epsilon_{0}, X_{1} \wedge X_{2} \wedge \ldots \wedge X_{m}(x)=0\right.$ or $\left.d f_{1} \wedge \ldots \wedge d f_{n-m}(x)=0\right\}$

the singular locus of our $n$-tuple of vector fields and functions $X_{1}, \ldots, X_{m}, f_{1}, \ldots$, $f_{n-m}$. By assumptions, $S$ is a complex analytic set of complex codimension at least 1. In particular, we have the following Łojasiewicz-type inequalities (see e.g. [6]): there exist a natural number $N$ and a positive constant $C$ such that

$$
\left|X_{1} \wedge \ldots \wedge X_{m}(x)\right| \geq C(d(x, S))^{N}
$$

and

$$
\left|d f_{1} \wedge \ldots \wedge d f_{n-m}(x)\right| \geq C(d(x, S))^{N}
$$

for any $x$ with $|x|<\epsilon_{0}$, where the norms applied to $X_{1} \wedge \ldots \wedge X_{m}(x)$ and $d f_{1} \wedge \ldots \wedge d f_{n-m}(x)$ are some standard norms in the space of $m$-vectors and $(n-m)$-vectors respectively, and $d(x, S)$ is the distance from $x$ to $S$ with respect to the Euclidean metric.

For each $d \in \mathbb{N}$ and a small positive number $\epsilon(d)>0$ (which will be chosen later in function of $d$, with $\lim _{d \rightarrow \infty} \epsilon(d)=0$ ), define the following open subset $U_{d, \epsilon(d)}$ of $\mathbb{C}^{n}$ :

$$
U_{d, \epsilon(d)}=\left\{x \in \mathbb{C}^{n},|x|<\epsilon(d), d(x, S)>|x|^{d}\right\} .
$$

For each $k=1, \ldots, r$, we will define a holomorphic vector field $\mathcal{Z}_{k}$ in $U_{d, \epsilon(d)}$, such that (the real part of) $\sqrt{-1} \mathcal{Z}_{k}$ is a periodic vector field of period $2 \pi$, and in such a way that for any two natural numbers $d_{1} \neq d_{2}$ the vector field $\mathcal{Z}_{k}$ defined for $U_{d_{1}, \epsilon\left(d_{1}\right)}$ coincides with the vector field (with the same name) $\mathcal{Z}_{k}$ defined for $U_{d_{2}, \epsilon\left(d_{2}\right)}$ on the intersection $U_{d_{1}, \epsilon\left(d_{1}\right)} \cap U_{d_{2}, \epsilon\left(d_{2}\right)}$, as follows.

Let

$$
\left(x_{j}^{d}\right)=\Phi^{D(d)}\left(x_{j}\right)=\left(x_{j}\right)+\text { higher order terms }
$$

be a local holomorphic coordinate transformation which puts $X$ into normal form up to order $D(d)$, where $D(d)$ is a sufficiently large number (as large as we 
wish), which will be chosen in function of $d$, with $\lim _{d \rightarrow \infty} D(d)=\infty$. We can choose $\Phi^{D(d)}$ in such a way that for any two natural numbers $d_{1} \neq d_{2}$ we have

$$
\Phi^{D\left(d_{1}\right)}\left(x_{j}\right)=\Phi^{D\left(d_{2}\right)}\left(x_{j}\right)+\text { terms of order } \geq \min \left(D\left(d_{1}\right), D\left(d_{2}\right)\right)
$$

Denote by

$$
Z_{k}^{d}=\sum_{j=1}^{n} \rho_{j}^{k} x_{j}^{d} \partial / \partial x_{j}^{d}
$$

the vector fields that are defined as in Equation (2.12), but for the semisimple part of the linear part of $X$ with respect to the local coordinate system $\left(x_{j}^{d}\right)$. In particular, $\sqrt{-1} Z_{k}$ is a holomorphic periodic vector field of period $2 \pi$ for each $k=1, \ldots, r$. According to Lemma 3.1, we have

$$
\left[Z_{k}^{d}, X_{j}\right](x)=O\left(|x|^{D(d)}\right) \quad \forall j=1, \ldots, m,
$$

and

$$
Z_{j}^{d}(\mathbf{f})(x)=O\left(|x|^{D(d)}\right)
$$

where $\mathbf{f}=\left(f_{1}, \ldots, f_{n-m}\right)$ denotes the vector-valued common first integral of $X_{1}, \ldots, X_{m}$. In other words, $\mathbf{f}$ is a vector-valued first integral of $Z_{k}^{d}$ up to order $D(d)$, and $X_{1}, \ldots, X_{m}$ commute with $Z_{j}^{d}$ up to order $D(d)$.

Let $y$ be an arbitrary point in $U_{d, \epsilon(d)}$. Then, due to the Lojasiewicz-type inequalities and the definition of $U_{d, \epsilon(d)}$, we have:

$$
\left|X_{1} \wedge \ldots \wedge X_{m}(y)\right|>C|y|^{d N}
$$

and

$$
\left|d f_{1} \wedge \ldots \wedge d f_{n-m}(y)\right|>C|y|^{d N} .
$$

Denote by $\Gamma_{k}^{d}(t)=\Gamma_{k}^{d}(t, y)$ the closed curve $(t \in[0,2 \pi])$ which is the orbit of the periodic vector field $\Re\left(\sqrt{-1} Z_{k}^{d}\right)$ which begins at $y$. We have that $\Gamma_{k}^{d}(0)=y$, and $\frac{1}{2}|y| \leq\left|\Gamma_{k}^{d}(t)\right| \leq 2|y|$ for any $t \in[0,2 \pi]$, provided that $\epsilon(d)$ is small enough.

It follows from the fact that $\mathbf{f}$ is a vector-valued first integral of $Z_{k}^{d}$ up to order $D(d)$ and $X_{1}, \ldots, X_{m}$ commute with $Z_{k}^{d}$ up to order $D(d)$, that we have the following inequalities, provided that $D(d)$ is large enough (say $D(d)>4 d N$ ) and $\epsilon(d)$ small enough:

$$
\begin{aligned}
& |\mathbf{f}(z)-\mathbf{f}(y)|<|y|^{D_{1}(d)} \\
& \left|\left[X_{j}, Z_{k}^{d}\right](z)\right|<|y|^{D_{1}(d)} \quad \forall j=1, \ldots, m \\
& \left|X_{1} \wedge \ldots \wedge X_{m}(z)\right|>\frac{C}{2}|y|^{d N} \\
& \left|d f_{1} \wedge \ldots \wedge d f_{n-m}(z)\right|>\frac{C}{2}|y|^{d N}
\end{aligned}
$$

for any point $z$ lying on the curve $\Gamma_{k}^{d}$. Here $D_{1}(d)$ is some sufficiently large number to be chosen in function of $d$. (We may choose $D_{1}(d)$ as large as we need, and then choose $D(d)$ and $\epsilon(d)$ correspondingly so that the above inequalities hold).

The above inequalities imply the following things: 
a) The curve $\Gamma_{k}^{d}$ is very close to the (regular part of the) level set $L_{y}=$ $\mathbf{f}^{-1}(\mathbf{f}(y))$ of the vector-valued first integral $\mathbf{f}=\left(f_{1}, \ldots, f_{n-m}\right)$ which contains the point $y$, in the sense that it can be projected orthogonally to a smooth closed curve $\hat{\Gamma}_{k}^{d}(t)(t \in[1,2 \pi])$ lying on $L_{y}$ which is very close to $\Gamma_{k}^{d}$ in $C^{1}$ topology: the distance from $\hat{\Gamma}_{k}^{d}$ to $\Gamma_{k}^{d}$ in $C^{1}$-topology is bounded from above by $|y|^{D_{2}(d)}$, where $D_{2}(d)$ is a sufficiently large number depending on $d$. (We may take $D_{2}(d)$ as large as we want, and then choose $D_{1}(d)$ and $\epsilon(d)$ correspondingly so that the above upper bound holds true).

b) The regular part of the level set $L_{y}$ is of complex dimension $m$, and its tangent space at each point is spanned by the vectors $X_{1}, \ldots, X_{m}$. Moreover, the regular part of $L_{y}$ has a flat affine structure given by the vector fields $X_{1}, \ldots, X_{m}$, because these vector fields commute.

c) If we write $d \hat{\Gamma}_{k}^{d}(t) / d t=\sum_{j=1}^{m} \Re\left(a_{k}^{j}(t) X_{j}\left(\hat{\Gamma}_{k}^{d}(t)\right)\right)$, then the complex functions $a_{k}^{j}(t)$ are nearly constant in the sense that $\left|a_{k}^{j}(t)-a_{k}^{j}(0)\right| \leq|y|^{D_{3}(d)}$ for $t \in[1,2 \pi]$, where $D_{3}(d)$ is a sufficiently large number depending on $d$ (we may take $\left.D_{3}(d)=D_{2}(d)-1\right)$. This fact follows from the almost commutativity of the vector fields $X_{1}, \ldots, X_{m}$ with the vector field $Z_{k}^{d}$ (the second line of (3.12)), and the fact that $\left|d \hat{\Gamma}_{k}^{d}(t) / d t-\Re\left(\sqrt{-1} Z_{k}^{d}\left(\hat{\Gamma}_{k}^{d}(t)\right)\right)\right|<|y|^{D_{2}(d)}$ by the above point a).

d) By approximation (implicit function theorem), there exist complex numbers $a_{k}^{1}, \ldots, a_{k}^{m}$ such that $\left|a_{k}^{j}-a_{k}^{j}(0)\right|<|y|^{D_{3}(d)}$, and that the time- $2 \pi$ flow of the vector field $\sum_{j=1}^{m} a_{k}^{j} X_{j}$ on $L_{y}$ fixes point $y$. In other words, the real vector field $\Re\left(\sum a_{k}^{j} X_{j}\right)$ has a periodic orbit of period $2 \pi$ which passes via $y$, and this orbit is $C^{1}$-close to $\hat{\Gamma}_{k}^{d}(t, y)$.

e) Due to the flat affine structure of $L_{y}$, the numbers $a_{k}^{1}, \ldots, a_{k}^{m}$ are well defined, i.e. unique. And they don't depend on the choice of $y$ in $L_{y}$ (at least locally). We may consider $a_{k}^{1}, \ldots, a_{k}^{m}$ as functions of $y: a_{k}^{1}(y), \ldots, a_{k}^{m}(y)$. These are holomorphic functions (due to the holomorphic implicit function theorem) which are constant on the connected components in $U_{d, \epsilon(d)}$ of the level sets of the vector-valued function $\mathbf{f}$, and are uniformly bounded in $U_{d, \epsilon(d)}$ by 1 (provided that $\epsilon(d)$ is small enough).

Now define the vector field $\mathcal{Z}_{k}$ as follows:

$$
\mathcal{Z}_{k}(y)=-\sqrt{-1} \sum_{j=1}^{m} a_{k}^{j}(y) X_{j}(y)
$$

Then $\mathcal{Z}_{k}$ is a holomorphic vector field in $U_{d, \epsilon(d)}$ with the following remarkable properties (for each $k=1, \ldots, r$ ):

a) $\mathcal{Z}_{k}$ is uniformly bounded by 1 , and $\sqrt{-1} \mathcal{Z}_{k}$ is a periodic vector field of period $2 \pi$ (at least in some open subset of $U_{d, \epsilon(d)}$ ).

b) If $\mathcal{Z}_{k}$ is a vector field defined as above for $U_{d, \epsilon(d)}$, and $\mathcal{Z}_{k}^{\prime}$ is also a vector field defined as above but for $U_{d^{\prime}, \epsilon\left(d^{\prime}\right)}$ with $d^{\prime} \neq d$, then $\mathcal{Z}_{k}$ coincides with $\mathcal{Z}_{k}^{\prime}$ on the intersection of $U_{d, \epsilon(d)}$ with $U_{d^{\prime}, \epsilon\left(d^{\prime}\right)}$, due to the uniqueness of $a_{k}^{j}$. Indeed, the vector field $\mathcal{Z}_{k}$ commutes with the vector field $\mathcal{Z}_{k}^{\prime}$ on $U_{d, \epsilon(d)} \cap U_{d^{\prime}, \epsilon\left(d^{\prime}\right)}$ 
by construction, their difference $\mathcal{Z}_{k}-\mathcal{Z}_{k}^{\prime}$ is tangent to the level sets of $\mathbf{f}$ in $U_{d, \epsilon(d)} \cap U_{d^{\prime}, \epsilon\left(d^{\prime}\right)}$ and is a constant vector field with respect to the flat affine structure on each level set, and $\sqrt{-1}\left(\mathcal{Z}_{k}-\mathcal{Z}_{k}^{\prime}\right)$ is periodic of period at most $2 \pi$ there. But the coefficients of $\mathcal{Z}_{k}(y)-\mathcal{Z}_{k}^{\prime}(y)$ when written as a linear combination of $X_{1}, \ldots, X_{m}$ are bounded from above by $|y|^{\min \left(D_{3}(d), D_{3}\left(d^{\prime}\right)\right)}$, i.e. $\sqrt{-1}\left(\mathcal{Z}_{k}-\mathcal{Z}_{k}^{\prime}\right)$ is too small to be periodic of period $2 \pi$ unless it is zero. Thus we have $\mathcal{Z}_{k}=\mathcal{Z}_{k}^{\prime}$ in $U_{d, \epsilon(d)} \cap U_{d^{\prime}, \epsilon\left(d^{\prime}\right)}$.

In other words, we have defined $r$ bounded holomorphic vector fields $\mathcal{Z}_{1}, \ldots, \mathcal{Z}_{r}$ on $U=\bigcup_{d=1}^{\infty} U_{d, \epsilon(d)}$. These vector fields commute pairwise due to the flat structure of the level sets $L_{y}$ (they are constant on each $L_{y}$ with respect to the flat structure). And $\sqrt{-1} \mathcal{Z}_{1}, \ldots, \sqrt{-1} \mathcal{Z}_{r}$ are periodic of period $2 \pi$ (ar least in some open subset). The following lemma taken from [12] shows that $\mathcal{Z}_{1}, \ldots, \mathcal{Z}_{r}$ can be extended holomorphically in a neighborhood of 0 in $\mathbb{C}^{n}$, i.e. there are holomorphic vector fields in a neighborhood of 0 in $\mathbb{C}^{n}$ which coincide with them on $U$. Thus we found the generators for an effective torus action of dimension $r$ which preserves $X=X_{1}$ (and $X_{2}, \ldots, X_{m}$ as well). Due to our approximation process, the linear part of $\mathcal{Z}_{k}$ is the same as the linear part of $Z_{k}^{d}$ (for any $d$ ), i.e. the weights of our torus action are appropriate in the sense of proposition 2.1. Thus we can apply Proposition 2.1 to finish the proof of Theorem 1.1.

Lemma $3.2([12])$. Let $U=\bigcup_{d=1}^{\infty} U_{d}$, with $U_{d}=\left\{x \in \mathbb{C}^{n},|x|<\epsilon_{d}, d(x, S)>\right.$ $\left.|x|^{d}\right\}$, where $\left(\epsilon_{d}\right)$ is an arbitrary series of positive numbers and $S$ is a local proper complex analytic subset of $\mathbb{C}^{n}\left(\right.$ codim $\left._{\mathbb{C}} S \geq 1\right)$. Then any bounded holomorphic function in $U$ has a holomorphic extension in a neighborhood of 0 in $\mathbb{C}^{n}$.

The proof of the above lemma is given in [12], so we will not repeat it here.

As a corollary of Theorem 1.1, we get the following result about simultaneous Poincaré-Dulac normal forms for commuting vector fields:

Theorem 3.3. Any $m$-tuple of pairwise commuting analytic vector fields $X_{1}, \ldots, X_{m}$ in a neighborhood of 0 in $\mathbb{K}^{n}(n \geq m \geq 1)$, which are linear independent almost everywhere, and which have $(n-m)$ functionally independent common analytic first integrals, admits a simultaneous convergent Poincaré-Dulac normalization.

Proof. By assumptions, each of the vector fields $X_{1}, \ldots, X_{m}$ is integrable in the non-Hamiltonian sense, so the proof of Theorem 1.1 shows that for each of them there is a torus action whose linearization normalizes it. Moreover, by construction, all these torus actions commute with each other and preserve all the vector fields $X_{1}, \ldots, X_{m}$. Thus we can combine these torus actions to get one "big" torus action whose linearization will normalize $X_{1}, \ldots, X_{m}$ simultaneously.

\section{The isochore case}

A normalization of an isochore (i.e. volume-preserving) vector field in $\left(\mathbb{C}^{n}, 0\right)$ is a Poincaré-Dulac normalization $\tilde{x}=\phi(x)$ which preserves the volume form 
$d x_{1} \wedge \ldots \wedge d x_{n}$. It is not surprising that an isochore vector field always admits a formal normalization. Notice that in the isochore case, there is at least one resonance relation: the sum of the eigenvalues is zero. This relation implies that the vector fields $Z_{1}, \ldots, Z_{r}$ defined in (2.12) are also isochore. Naturally, Proposition 2.1 also has an isochore version.

Proposition 4.1. A holomorphic vector field $X$ in a neighborhood of 0 in $\mathbb{C}^{n}$ admits a convergent Poincaré-Dulac normalization if and only if it is preserved by an effective holomorphic isochore action of a real torus $\mathbb{T}^{r}$ in a neighborhood of 0 in $\mathbb{C}^{n}$, where $r$ is the toric degree of $X$, which has 0 as a fixed point and whose linear part at 0 has appropriate weights (given by the lattice $\mathcal{Q}$ defined in (2.9), which depends only on the linear part $X^{(1)}$ of $\left.X\right)$.

The proof of Proposition 4.1 is absolutely similar to the proof of Proposition 2.1 .

Similarly, Proposition 2.2 remains true in the isochore case as well.

In [11], Vey showed that if $n-1$ pairwise commuting isochore vector fields in $\mathbb{C}^{n}$ have linearly independent diagonalizable linear parts, then they are simultaneously normalizable. Though Vey's proof didn't use the fast convergence method directly, it used a result of Malgrange on "Frobenius with singularities" [7], which in turn had been proved by the fast convergence method. We will show that Vey's theorem is still true without any nondegeneracy condition:

Theorem 4.2. Any $(n-1)$-tuple of pairwise commuting analytic isochore vector fields in a neighborhood of 0 in $\mathbb{K}^{n}$, which are linearly independent almost everywhere, admits a simultaneous convergent normalization.

Note that in the above theorem, though the vector fields are assumed to be linearly independent almost everywhere, their linear parts may be very degenerate and may indeed be linearly dependent.

We will only give here a sketch of the proof of the above theorem. It consists of the following steps:

1) Find a first integral: The 1-form $\alpha=i_{X_{1}} i_{X_{2}} \ldots i_{X_{n-1}} \Omega$, where $\Omega$ is the volume form which is preserved by $(n-1)$ pairwise commuting vector fields $X_{1}, \ldots, X_{n-1}$, is closed, hence exact (Poincaré's lemma). If $g$ is a function such that $d g=\alpha$ then $g$ is a common first integral for our vector fields.

2) Use Theorem 3.3 , since now we have $n-1$ commuting vector fields and 1 first integral.

3) Keep track of the isochore condition. (Everything is made in an isochore way).

Details are left to the reader.

\section{Acknowledgement}

I would like to thank the referee for his critical remarks. 


\section{References}

1. D. Bambusi, G. Cicogna, G. Gaeta, G. Marmo, Normal forms, symmetry and linearization of dynamical systems, J. Phys. A 31 (1998), no. 22, 5065-5082.

2. O. I. Bogoyavlenskij, A concept of integrability of dynamical systems, C. R. Math. Rep. Acad. Sci. Canada, 18 (1996), no. 4, 163-168.

3. A. D. Bruno, Local methods in nonlinear differential equations, Springer Series in Soviet Mathematics, Springer-Verlag, Berlin, 1989.

4. A. D. Bruno, S. Walcher, Symmetries and convergence of normalizing transformations, J. Math. Anal. Appl. 183 (1994), no. 3, 571-576.

5. G. Cicogna, S. Walcher, Convergence of normal form transformations: The role of symmetries, to appear in Acta Appl. Math., no. 70 (2002).

6. S. Łojasiewicz, Sur le problème de la division, Studia Math. 18 (1959), 87-136.

7. B. Malgrange, Frobenius avec singularités. 1: Codimension un, Inst. Hautes Études Sci. Publ. Math., no. 46 (1976), 163-173.

8. R. Roussarie, Modèles locaux de champs et de formes, Astérisque, no. 30, Société Mathématique de France, Paris, 1975. 181 pp.

9. L. Stolovitch, Singular complete integrability, Inst. Hautes Études Sci. Publ. Math., no. 91 (2000), 133-210 (2001).

10. L. Stolovitch, Normalisation holomorphe d'algèbres de type Cartan de champs de vecteurs holomorphes singuliers, C. R. Acad. Sci. Paris Sér. I Math. 330 (2000), no. 2, 121-124.

11. J. Vey, Algèbres commutatives de champs de vecteurs isochores, Bull. Soc. Math. France, 107 (1979), no. 4, 423-432.

12. Nguyen Tien Zung, Convergence versus integrability in Birkhoff normal form, preprint: math.DS/0104279

13. Nguyen Tien Zung, Reduction and integrability, preprint: math.DS/0201087

GTA, UMR 5030 CNRS, DÉPartement de Mathématiques, Université Montpellier II, FRANCE.

E-mail address: tienzung@math.univ-montp2.fr 